\title{
Burkitt Lymphoma in Children: An Unusual Presentation
}

\section{Carmela Brillantino $^{1}$ | Eugenio Rossi $^{1}$ | Rocco Minelli2* | Delfina Bifano ${ }^{3}$ | Elio Bignardi ${ }^{4}$ Raffaele Zeccolini $^{5}$ | Massimo Zeccolini6}

*Correspondence: Rocco Minelli

Address: ${ }^{1}$ Unit of Radiology and Ultrasound, A.O.R.N. Santobono-Pausilipon, Pausilipon Hospital, Naples, Italy; ${ }^{2}$ Department Life and Health "V. Tiberio", University of Molise, Campobasso, Italy; ${ }^{3}$ Unit of Pathological Anatomy, A.O.R.N. SantobonoPausilipon, Pausilipon Hospital, Naples, Italy; ${ }^{4}$ Unit of Imaging Diagnostics, A.O.R.N. dei Colli, Cotugno Hospital, Naples, Italy; ${ }^{5}$ Luigi Vanvitelli University, Naples, Italy; ${ }^{6}$ Unit of Radiology, A.O.R.N. Santobono-Pausilipon, Santobono Hospital, Naples, Italy e-mail $\bowtie$ : rocco.minelli@libero.it

Received: 12 May 2020; Accepted: 01 June 2020

Copyright: (C) 2020 Brillantino C. This is an open-access article distributed under the terms of the Creative Commons Attribution License, which permits unrestricted use, distribution, and reproduction in any medium, provided that the original work is properly cited.

\section{ABSTRACT}

Burkitt lymphoma is an extremely aggressive and fast-growing mature B-cell non-Hodgkin lymphoma. There are three clinical variants of Burkitt's lymphoma: endemic or African, sporadic and related to immunodeficiency. The sporadic variant of Burkitt's lymphoma is mainly present in Western countries and represents $40 \%$ of all childhood non-Hodgkin lymphomas. The average age of onset is 8 years. In pediatric age, sporadic Burkitt lymphoma is mainly an extranodal disease and at diagnosis the most commonly involved site is the gastrointestinal system, in the form of a massive abdominal mass occupying the lower right quadrant and which arises in the region of the ileocecal valve. Sporadic Burkitt lymphoma occurs less frequently with cervical lymphadenopathies, and other organs such as the brain, lung, liver, spleen, kidney and bone are commonly affected in the systemic spread of the tumor. In this article we describe an unusual presentation of advanced LB. A 5-year-old boy came to our observation due to a left submandibular lymph node swelling that was biopsied with the diagnosis of LB. Subsequently, radiological investigations performed for staging unexpectedly showed liver, kidney and brain injuries.

Keywords: Burkitt Lymphoma, Meningeal Infiltrates, Ultrasound, CT, MRI

\section{Introduction}

Burkitt lymphoma (LB) is an extremely aggressive mature B-cell non-Hodgkin lymphoma characterized by very fast growth with a duplication time of 24-48 hours (disease (Abramson and Price, 2008; Derinkuyu et al., 2016). The LB can be divided into three clinical variants: the endemic that occurs in equatorial Africa, the sporadic present in Western countries, and the variant related to immunodeficiency. The sporadic variant of LB represents $40 \%$ of all childhood non-Hodgkin lymphomas (Abramson and Price, 2008; Derinkuyu et al., 2016; Kalisz et al., 2019). The average age of onset is 8 years and males are more affected than females (Abramson and Price, 2008; Derinkuyu et al., 2016; Kalisz et al., 2019; Toma et al., 2007). In children, sporadic LB is predominantly an extranodal abdominal-onset disease; most children have a mass in the lower right quadrant of the abdomen that may involve the terminal ileum, the blind, the ascending colon or the appendix (Abramson and Price, 
2008; Derinkuyu et al., 2016; Kalisz et al., 2019; Toma et al., 2007). Sporadic LB occurs less frequently with large cervical adenopathies (Abramson and Price, 2008; Derinkuyu et al., 2016; Kalisz et al., 2019). Primary involvement of the brain, lung, liver, spleen, kidney and bone occurs rarely and lesions of these organs are usually manifestations of widespread disease (Abramson and Price, 2008; Derinkuyu et al., 2016; Kalisz et al., 2019; Toma et al., 2007).

\section{Case Report}

In February 2020, an asymptomatic 5-year-old boy underwent a pediatric examination for a left submandibular swelling that gradually increased in size, with a hard-elastic consistency and was not painful on palpation. Blood tests, prescribed by the pediatrician and performed in a private medical center, were normal: the blood count with the leukocyte formula and the inflammation indexes such as ESR and PCR were not altered, LDH was normal and anti-CMV IgM were negative, anti-EBV VCA and anti-toxoplasma IgM. The child underwent an ultrasound of the neck which showed numerous lymph nodes increased in size in the left submandibular site; the largest lymph node measured approximately 27x16 mm and showed a colliqued aspect. The pediatrician prescribed amoxicillin / clavulanic acid therapy for six days and despite taking antibiotics, the swelling had increased in size. In March the boy came to our hospital for further diagnostic information; he repeated the blood tests, which were normal, and underwent an ultrasound of the cervical, axillary and inguinal lymph nodes, testicles and complete abdomen. The ultrasound of the neck showed in the left submandibular seat a coarse lymph node of the size of $38 \times 30 \mathrm{~mm}$, with a markedly and unevenly hypoechoic echostructure and anarchic vascularization at the color-Doppler (Fig. 1).

Other lymph nodes with similar ultrasound features, but of a smaller size, were evident in the same site. There were no pathological lymph nodes in the other cervical lymphatic stations, in the axillary and inguinal site bilaterally and the ultrasound examination of the testicles was negative. The ultrasound of the complete abdomen showed liver and kidneys with an echo structure widely subverted due to the presence of multiple hypoechoic nodular areas; these lesions, of a maximum size not exceeding $4 \mathrm{~cm}$, did not show vascular flows internal to the color-Doppler (Fig. 2).

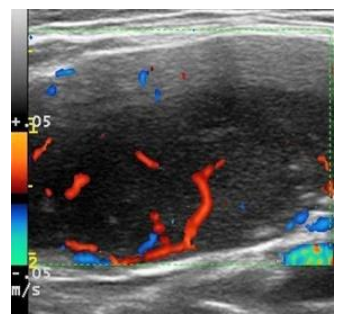

Figure 1: Longitudinal ultrasound scan of the left submandibular swelling shows a lymphadenopathy with markedly hypoechoic echotexture and anarchic vascularization on color-Doppler 

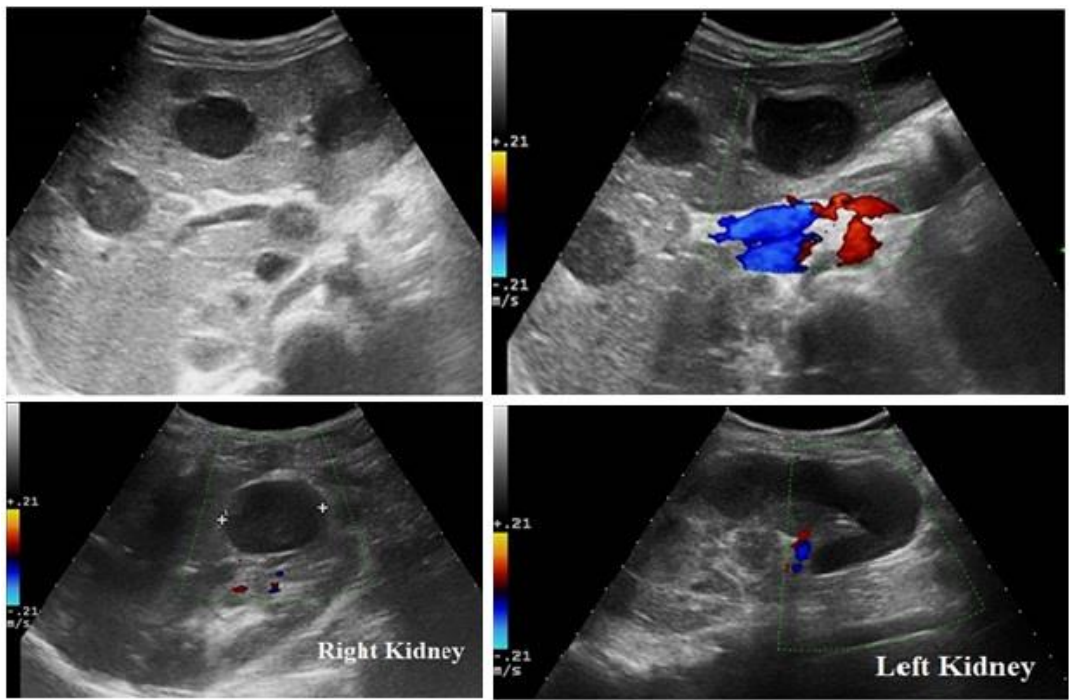

Figure 2: Abdominal ultrasound shows numerous hypoechoic lesions in the hepatic $(a, b)$ and renal parenchyma (c, d), without detectable vascularization at color-Doppler (b, c, d)

The child was then subjected to a surgical biopsy of the left submandibular lymph node swelling with extensive removal of tissue intended for histological examination. The microscopic analysis revealed hypercellular fragments consisting of a widespread and monomorphic proliferation of atypical lymphoid cells mainly of medium size with poor cytoplasm and roundish nucleus provided with one or more nucleoli; numerous apoptotic cells and many dyeable body macrophages were present; mitotic figures were frequently observed and the proliferation index (Ki67) was approximately 100\%. In the immunohistochemical investigation, the cells showed positivity for CD45, CD20, CD79, PAX-5, TCL-1, BCL6, CD10, CD43 and CD38; in addition, 80\% of the cell population expressed the nuclear protein cmyc (Fig. 3).

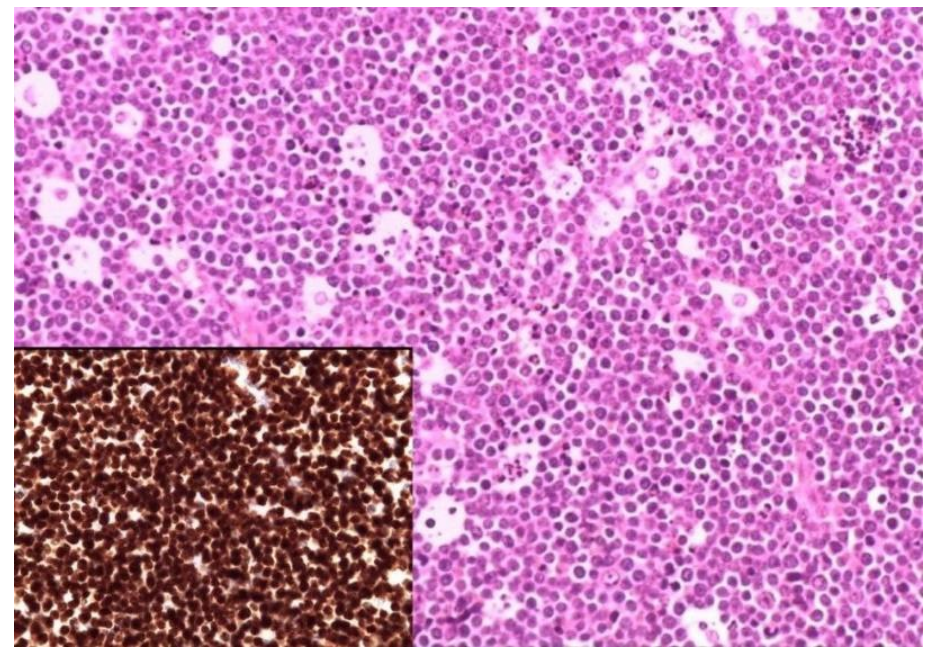

Figure 3: Classic Burkitt Lymphoma. Typical starry sky pattern due to the presence of numerous phagocytic histiocytes engulfing nuclear debris; cytologic features with relatively uniform round or oval nuclei, multiple small nucleoli, and thin rims of cytoplasm. EE 200X. INSET: The cells show a 100\% of Ki67 staining.400X 
The histological finding was compatible with LB and the child underwent a total body CT with contrast medium for staging. The CT scan of the skull showed meningeal thickenings from infiltration of the temporal pole bilaterally, along the free margin of the cavernous sinus tentorium and in the left parieto-occipital site (Fig. 4).
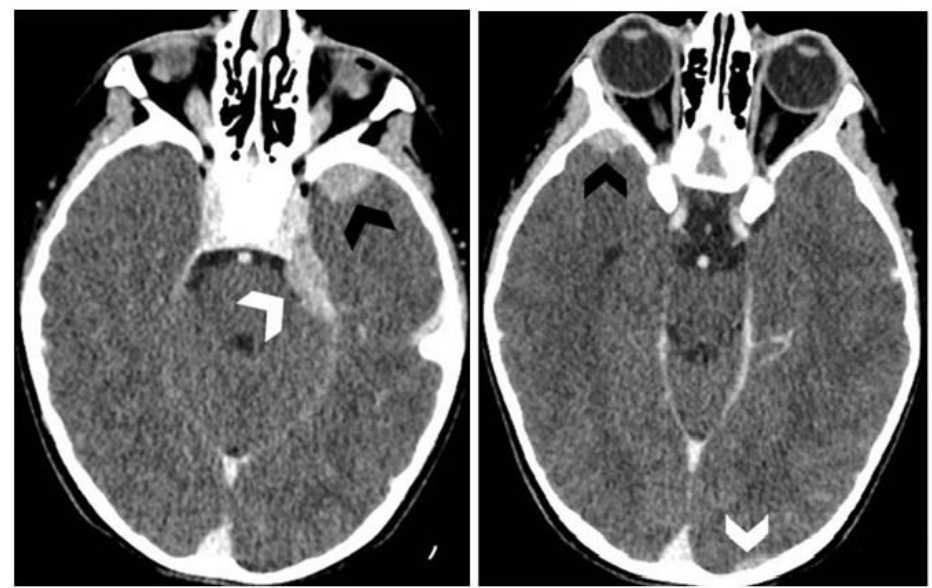

Figure 4: AXIAL POST-CONTRAST CT IMAGES OF THE HEAD (A, B) SHOW enhancing meningeal infiltrates located on the left in the temporal pole (a, black arrowhead) and along the free edge of the tentorium-cavernous sinus (a, white arrowhead), and placed in the right temporal pole (b, black arrowhead) and left parieto-occipital region (b, white arrowhead)

Chest CT was negative and CT of the neck and full abdomen showed left submandibular lymphadenopathy and multiple liver and kidney lesions that appeared hypodense, respectively (Fig. 5).
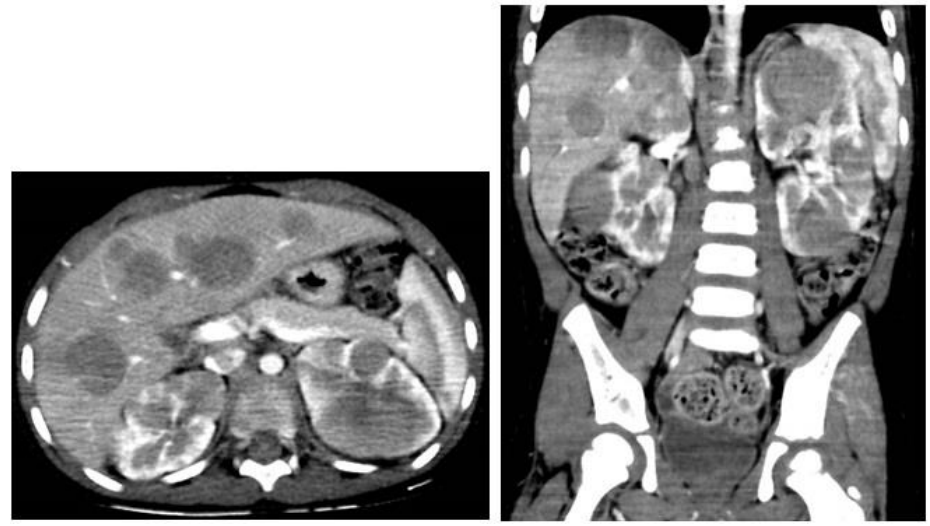

Figure 5: AXIAL (A) AND CORONAL (B) POST-CONTRAST CT IMAGES OF THE ABDOMEN show multiple low-attenuation lesions with minimal enhancement in liver and in both kidneys

On the basis of the brain findings, the child was subjected to an examination of the cerebrospinal fluid and MRI of the brain and spine. The analysis of the CSF showed a small percentage (1\%) of lymphoid cells with pathological B phenotype. MRI of the brain showed the lesions reported on the CT examination, but also the perineural diffusion of the LB showing the swelling and the altered signal intensity of the left trigeminal nervein the prepontine cistern and the Meckel cable (Fig. 6). 

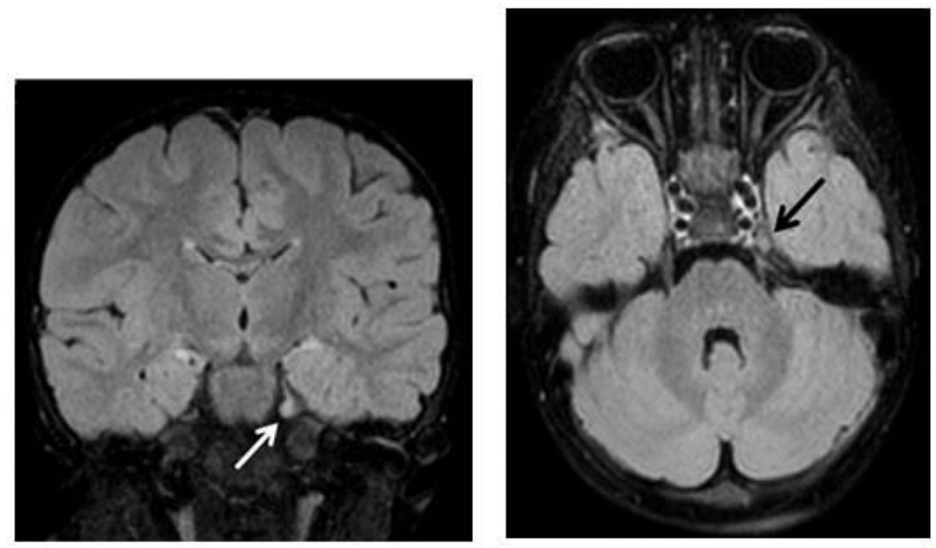

Figure 6: Coronal (a) and axial (b) FLAIR MR images show marked thickening of the left trigeminal nerve in the prepontine cistern (a, white arrow) and Meckel's cave (b, black arrow)

Finally, there was no spinal spread of the disease on MRI of the spine. Once staging was complete, the child began specific treatment for the treatment of LB.

\section{Discussion}

LB is an aggressive neoplasm that mainly affects male children under 10 years of age and which has the abdomen as the most frequent localization (Abramson and Price, 2008; Derinkuyu et al., 2016; Kalisz et al., 2019; Toma et al., 2007; Brillantino et al., 2020). In about 20-30\% of cases the head-neck region, which includes the Waldeyer ring or cervical lymph nodes, is the site of origin of the disease and the most frequent onset symptom is a superficial swelling of the painless neck resulting from the confluence of multiple lymph nodes increased in size (Derinkuyu et al., 2016; Kalisz et al., 2019; Huang et al., 2010; Lloyd and McHugh, 2010). The ultrasound is the first choice radiological investigation for the initial evaluation of a cervical swelling; although it is an operator-dependent imaging method, it is not invasive and is therefore easily executable and repeatable, does not require exposure to ionizing radiation, has a high diagnostic sensitivity and analyzes the vascular flow through the Doppler effect (Derinkuyu et al., 2016; Kalisz et al., 2019; Toma et al., 2007; Lloyd and McHugh, 2010; Caprio et al., 2019). The ultrasound assesses the origin of cervical swelling, confirming or excluding lymphodenopathy, and plays an important role in the differential diagnosis between inflammatory lymph nodes, very frequent in children, and neoplastic. No single ultrasound feature of lymphadenopathy is predictive of benignity or malignancy, but the simultaneous presence of multiple ultrasound findings such as: rounded shape, hypoechoic ecostructure, absent or eccentric hyperechoic ile, increased and chaotic vascularization, makes the diagnosis of malignancy highly probable (Kalisz et al., 2019; Toma et al., 2007; Lloyd and McHugh, 2010; Caprio et al., 2019; Pessey et al., 2009). In our case the ultrasound features of the left submandibular lymphadenopathy suggested a possible neoplastic etiology also in consideration of the elements (Lloyd and McHugh, 2010). On the other hand, 
on ultrasound of the abdomen, the finding of suspected repetitive lesions in the liver and renal parenchyma, suggested a rapidly progressing neoplasm. In particular, given the age of the child, we believed the presence of LB to be very likely, which is often already disseminated when it occurs with involvement of the cervical lymph nodes (Toma et al., 2007; Lloyd and McHugh, 2010; Mukhtar et al., 2017). In fact, in LB liver and kidney lesions are usually secondary, occur in $17 \%$ and $30 \%$ of cases respectively, and on ultrasound they can occur as multiple nodulations or hypoechoic masses, as happened in our case (Derinkuyu et al., 2016; Kalisz et al., 2019; Toma et al., 2007; Biko et al., 2009; Di Serafino et al., 2020). The histological analysis of the biopsy fragments of the left submandibular lymphadenopathy confirmed the presence of the LB and demonstrated the great utility and high diagnostic accuracy of the ultrasound. Once the diagnosis was established, the child underwent a total body CT examination with contrast medium. for staging and evaluating other possible disease sites. Unfortunately, CT scan of the skull showed meningeal infiltrates predicting poor prognosis. LB rarely occurs at diagnosis with central nervous system involvement which is observed in $12 \%$ of patients and is usually the expression of advanced disease (Abramson and Price, 2008; Derinkuyu et al., 2016; Kalisz et al., 2019; Toma et al., 2007; Huang et al., 2010). MRI of the brain highlighted the lesions described on the CT examination and thanks to the high spatial resolution and the intrinsic contrast resolution, it also diagnosed the perineural infiltration of the LB, showing the thickening and the altered signal strength of the nerve left trigeminal in the prepontine cistern and in the Meckel cable (Derinkuyu et al., 2016; Kalisz et al., 2019; Toma et al., 2007; Camilo et al., 2014; Malhotra et al., 2018).

\section{Conclusion}

In children, the finding of cervical lymph node swelling is extremely frequent; in most cases it is the response to infections of the upper respiratory-digestive tract, however it can also be the expression of a severe prognosis pathology, as has happened in our case. Therefore, children with cervical lymphadenopathy need a correct clinical-laboratory and instrumental framework in order to quickly reach an etiological diagnosis and detect the presence of a malignant neoplasm.

Funding Sources: This research did not receive any specific grant from funding agencies in the public, commercial, or not-for-profit sectors.

Declaration of Competing Interest: There are no conflicts of interest to disclose.

Acknowledgements: None. 


\section{References}

Abramson SJ and Price AP. Imaging of pediatric lymphomas. Radiol Clin North Am 2008; 46: 313-338.

Biko DM, Anupindi SA, Hernandez A, Kersun L, Bellah R. Childhood Burkitt lymphoma: abdominal and pelvic imaging findings. AJR Am J Roentgenol 2009; 192: 1304-1315.

Brillantino C, Rossi E, Bifano D, Minelli R, Tamasi S, Mamone R, Bignardi E, Zeccolini R, Zeccolini M, Vallone G. An unusual onset of pediatric acute lymphoblastic leukemia. J Ultrasound 2020; 23: 1-6

Camilo GB, Machado DC, de Oliveira CE, Lacerda Lda S, de Oliveira RV, Silva Mde F, Lopes AJ. Burkitt lymphoma with initial clinical presentation due to infiltration of the central nervous system and eye orbits. Am J Case Rep 2014; 15: 404-410.

Caprio MG, Di Serafino M, Pontillo G, Vezzali N, Rossi E, Esposito F, Zeccolini M, Vallone G. Paediatric neck ultrasonography: a pictorial essay. J Ultrasound 2019; 22: 215-226.

Derinkuyu BE, Boyunağa Ö, Öztunalı Ç, Tekkeşin F, Damar Ç, Alımlı AG, Okur A. Imaging features of Burkitt lymphoma in pediatric patients. Diagn Interv Radiol 2016; 22: 95-100.

Di Serafino M, Severino R, Gioioso M, Rossi E, Vezzali N, Pelliccia P, Caprio MG, Acampora C, Iorio R, Vallone G. Paediatric liver ultrasound: a pictorial essay. J Ultrasound 2020; 23: 87-103.

Huang MS, Tarbell NJ, Weinstein HJ. Chapter 8. p. 167: Non-Hodgkin Lymphoma. In: Halperin EC, Constine LS, Tarbell NJ, Kun LE, editors. Pediatric Radiation Oncology. 5th ed. Philadelphia: Lippincott Williams and Wilkins Publishers, 2010.

Kalisz K, Alessandrino F, Beck R, Smith D, Kikano E, Ramaiya NH, Tirumani SH. An update on Burkitt lymphoma: a review of pathogenesis and multimodality imaging assessment of disease presentation, treatment response, and recurrence. Insights Imaging 2019; 10: 56.

Lloyd C and McHugh K. The role of radiology in head and neck tumours in children. Cancer Imaging 2010; 10 : 49-61.

Malhotra A, Tu L, Kalra VB, Wu X, Mian A, Mangla R, Michaelides E, Sanelli P, Gandhi D. Neuroimaging of Meckel's cave in normal and disease conditions. Insights Imaging 2018; 9: 499-510.

Mukhtar F, Boffetta P, Risch HA, Park JY, Bubu OM, Womack L, Tran TV, Zgibor JC, Luu HN. Survival predictors of Burkitt's lymphoma in children, adults and elderly in the United States during 2000-2013. Int J Cancer 2017; 140: 1494-1502.

Pessey JJ, Rose X, Vergez S. Cervical adenopathies. EMC-Otolaryngology 2009; 8: 1-14.

Toma P, Granata C, Rossi A, Garaventa A. Multimodality Imaging of Hodgkin Disease and Non-Hodgkin Lymphomas in Children. Radiographics 2007; 27: 1335-1354. 